\title{
MicroRNA-148a inhibits cell proliferation and cell cycle progression in lung adenocarcinoma via directly targeting transcription factor E2F3
}

\author{
JIANWEI LIU ${ }^{1,2^{*}}$, LIBO SI $^{1 *}$ and HUI TIAN ${ }^{1}$ \\ ${ }^{1}$ Department of Thoracic Surgery, Qilu Hospital of Shandong University, Jinan, Shandong 250012; \\ ${ }^{2}$ Department of Thoracic Surgery, Affiliated Hospital of Binzhou Medical College, Binzhou, Shandong 256600, P.R. China
}

Received March 20, 2018; Accepted September 24, 2018

DOI: $10.3892 /$ etm.2018.6845

\begin{abstract}
MicroRNAs (miRs) serve important roles in various human cancers, including lung adenocarcinoma. Exploring the function and regulatory mechanism of miRs underlying lung adenocarcinoma progression may contribute to identifying novel therapeutic targets and candidates. The present study aimed to examine miR-148a expression and investigate the molecular mechanisms of miR-148a in lung adenocarcinomas. The data from the current study indicated that miR-148a was significantly downregulated in lung adenocarcinoma tissues and cell lines, and low miR-148a expression was significantly associated with advanced Tumor, Node, Metastasis stages and lymph node metastasis, as well as the shorter survival time of patients. Increased miR-148a expression markedly decreased the cell proliferation, colony formation and cell cycle progression of H23 and H1975 cells. Transcription factor E2F3 (E2F3) was identified as a target of miR-148a in H23 and H1975 cells. The expression of E2F3 was negatively mediated by miR-148a in H23 and H1975 cells. In addition, E2F3 was significantly upregulated in lung adenocarcinoma tissues and cell lines, and the expression of miR-148a was inversely correlated with E2F3 expression in lung adenocarcinoma tissues. Additional experiments demonstrated that increased E2F3 expression counteracted the inhibitory effects on lung adenocarcinoma cells caused by miR-148a overexpression. In summary, the findings of the current study suggest that miR-148a may have suppressive effects on the proliferation of lung adenocarcinoma cells at least in part through directly targeting E2F3.
\end{abstract}

Correspondence to: Professor Hui Tian, Department of Thoracic Surgery, Qilu Hospital of Shandong University, 107 Wenhuaxi Road, Jinan, Shandong 250012, P.R. China

E-mail: tianhuidoctor@aliyun.com

${ }^{*}$ Contributed equally

Key words: microRNA-148a, tumour suppressor, transcription factor E2F3, lung adenocarcinoma
Therefore, miR-148a may be used as a potential candidate for the treatment of lung adenocarcinoma.

\section{Introduction}

Lung cancer is the most common cancer type in men and women worldwide, causing a large number of cancer-associated mortalities $(1,2)$. Lung adenocarcinoma constitutes $\sim 50 \%$ of lung cancer cases worldwide $(1,2)$. Despite advances in the treatment of lung adenocarcinoma in recent decades, the overall 5-year survival rate of lung adenocarcinoma remains poor, mainly due to the poor understanding of lung adenocarcinoma pathogenesis (1). Exploring the molecular mechanisms underlying lung adenocarcinoma progression may assist in the development of novel therapeutic strategies.

MicroRNAs (miRs) are a type of small non-coding RNA containing 22-25 nucleotides and are key regulators of gene expression by directly interacting with the 3 '-untranslated regions (3'-UTRs) of their target mRNAs, leading to mRNA degradation or translation inhibition $(3,4)$. A large number of miRs are associated with the regulation of various biological processes, including cell proliferation, differentiation, apoptosis and migration, and cell cycle progression (4). Additionally, through mediating the expression of oncogenes or tumour suppressors, numerous miRs have been revealed to serve suppressive or promoting roles in the initiation and malignant progression of human cancers including lung adenocarcinoma (5-8). Exploring the expression and function of miRs in lung adenocarcinoma may help identify novel biomarkers and therapeutic targets for this disease.

Recently, miR-148a has been reported to be aberrantly expressed in different human cancers and serve tumour suppressive or promoting roles (9-11). For instance, miR-148a expression was significantly downregulated in gastric cancer tissues and cell lines, and served as a tumour suppressorin gastric carcinogenesis by inactivating signal transducer and activator of transcription (STAT) 3 and RAC- $\alpha$ serine/threonine-protein kinase, thus targeting gastrin/cholecystokinin type $\mathrm{B}$ receptor (CCK-BR) (9). Recently, Li et al (12) reported that the expression of miR-148 was significantly lower in the serum of patients with non-small-cell lung cancer (NSCLC) compared with that of patients with benign pulmonary diseases and 
healthy controls. Additionally, miR-148a has been reported to serve a suppressive role in NSCLC (13-15). However, the regulatory mechanism of miR-148a in lung adenocarcinoma growth remains largely unclear. The present study aimed to explore the clinical significance of miR-148a expression in lung adenocarcinoma, and investigate its function and regulatory mechanisms in lung adenocarcinoma cell proliferation.

\section{Materials and methods}

Tissue samples. The current study was approved by the Ethics Committee of the Affiliated Hospital of Binzhou Medical College (Binzhou, China). Lung adenocarcinoma and adjacent non-tumour tissues were collected from 53 patients with lung adenocarcinoma between June 2011 and October 2013 at Affiliated Hospital of Binzhou Medical College. Table I summarizes the clinical and pathological features of these patients. Written informed consent was obtained from all patients, and none of the patients underwent chemotherapy or radiotherapy prior to surgery. All tissues were quickly snap-frozen in liquid nitrogen following surgical resection and stored at $-80^{\circ} \mathrm{C}$ prior to use.

Cell culture. Several common lung adenocarcinoma cell lines (H23, H1975, H2228, and H2085) and a normal bronchial epithelium cell line (BEAS-2B) were obtained from American Type Culture Collection (Manassas, VA, USA). These cells were cultured in Dulbecco's modified Eagle's medium (DMEM) containing 10\% foetal bovine serum (FBS; both Thermo Fisher Scientific, Inc., Waltham, MA, USA). Cells were all maintained in a humidified incubator containing $5 \% \mathrm{CO}_{2}$ and $95 \% \mathrm{O}_{2}$ at $37^{\circ} \mathrm{C}$.

Cell transfection. Cells transfection was conducted using Lipofectamine 2000 (Thermo Fisher Scientific, Inc.) following the manufacturer's protocol. $100 \mathrm{nM}$ miR-148a mimics (cat. no. HmiR0102-MR04), negative control (NC) miR mimics (miR-NC; cat. no. CmiR0001-MR04), miR-148a inhibitor (cat. no. HmiR-AN0204-SN-10), NC inhibitor (cat. no. CmiR-AN0001-SN; all Guangzhou Fulengen Co., Ltd., Guangzhou, China), or $1 \mathrm{mg}$ pcDNA3.1-E2F3 expression plasmid or blank pcDNA3.1 plasmid (both Hunan Nanhua Aishi Pulin Biotechnology; NanHua Bio-medicine Co., Ltd., Changsha, China) were transfected into H23 and H1975 cells ( $5 \times 10^{6}$ cells/well). Following transfection for $48 \mathrm{~h}$, subsequent experiments were conducted.

Reverse transcription-quantitative polymerase chain reaction $(R T-q P C R)$. Total RNA was extracted from tissues and cell lines using TRIzol Reagent (Thermo Fisher Scientific, Inc.) and then reverse transcribed into cDNA using SuperScript III Reverse Transcriptase (Thermo Fisher Scientific, Inc.), according to the manufacturer's protocol. qPCR was conducted using the SYBR-Green Real-Time Master mix (Toyobo Life Science, Osaka, Japan) according to the manufacturer's protocol. The reaction was performed under the following conditions: $95^{\circ} \mathrm{C}$ for $5 \mathrm{~min}$, and 40 cycles at $95^{\circ} \mathrm{C}$ for $15 \mathrm{sec}$ and $60^{\circ} \mathrm{C}$ for $15 \mathrm{sec}$. GAPDH and U6 were used as internal controls to normalise the expression of E2F3 and miR-148a, respectively. The relative expression was calculated by the $2^{-\Delta \Delta \mathrm{Cq}}$ method (16). The primer sequences were as follows: E2F3 forward, 5'-AGAAAG CGGTCATCAGTACCT-3' and reverse, 5'-TGGACTTCGTAG TGCAGCTCT-3'; GAPDH forward, 5'-GGAGCGAGATCC CTCCAAAAT-3' and reverse, 5'-GGCTGTTGTCATACT TCTCATGG-3'; U6 forward, 5'-CTCGCTTCGGCAGCA CATATACT-3' and reverse, 5'-CGCTTCACGAATTTGCGT GT-3'. In addition, the primers for miR-148a were purchased from Guangzhou Fulengen Co., Ltd. (cat. no. HmiRQP0204) and the sequences were not supplied by the manufacturer. The experiments were repeated three times.

Cell proliferation analysis. Following transfection, the $\mathrm{H} 23$ and $\mathrm{H} 1975$ cells were seeded onto 96 -well plates at a density of $2 \times 10^{3}$ cells/well and cultured in DMEM supplemented with $10 \% \mathrm{FBS}$ at $37^{\circ} \mathrm{C}$ for $0,24,48$ and $72 \mathrm{~h}$. Cell proliferation was determined using Cell Counting kit-8 (Thermo Fisher Scientific, Inc.), according to the manufacturer's protocol. Absorbance was detected at an optical density of $450 \mathrm{~nm}$ by a spectrophotometer. The experiments were repeated three times.

Cell cycle analysis. Transfected H23 and H1975 cells were washed twice with ice-cold PBS and fixed with $70 \%$ ethanol at $-20^{\circ} \mathrm{C}$ overnight. Cells were incubated with $50 \mathrm{mg} / \mathrm{ml}$ propidium iodide (Thermo Fisher Scientific, Inc.) and $1 \mathrm{mg} / \mathrm{ml}$ RNase for $30 \mathrm{~min}$ in the dark at room temperature. The distribution of the cell cycle was analysed using a flow cytometer and BD Accuri $^{\mathrm{TM}}$ C6 software (version 1.0; BD Biosciences, Franklin Lakes, NJ, USA). The experiments were repeated three times.

Colony formation assay. Transfected H23 and H1975 cell suspensions (300 cells/well) were seeded in a 6-well plate and cultured in a humidified incubator containing $5 \% \mathrm{CO}_{2}$ at $37^{\circ} \mathrm{C}$ for 2 weeks. Then, the cells were fixed with $4 \%$ polyformaldehyde at room temperature for $30 \mathrm{~min}$ and stained with $0.1 \%$ crystal violet at room temperature for $10 \mathrm{~min}$. The number of colonies was counted under a light microscope (magnification, x10). The experiments were repeated three times.

Bioinformatics analysis and dual-luciferase reporter gene assay. TargetScan 7.1 software (http://www.targetscan.org/) was used to predict the putative targets of miR-148a. The wild type (WT) and mutant type (MT) sequences in the 3'-UTR of E2F3 mRNA, which contained the putative binding sites of miR-148a, were cloned into the pMIR-REPORT luciferase reporter plasmids (Promega Corporation, Madison, WI, USA). Transfected H23 and H1975 cells were then transfected with miR-148a mimics or miR-NC with WT E2F3 or MT E2F3 plasmids, respectively, using Lipofectamine 2000. After the cells were transfected for $48 \mathrm{~h}$, the relative luciferase activity was determined using the Dual-Luciferase ${ }^{\circledR}$ Reporter Assay system (Promega Corporation) according to the manufacturer's protocol. The activity of firefly luciferase was normalized to the activity of Renilla luciferase. The experiments were repeated three times.

Western blot analysis. Total protein was isolated from $\mathrm{H} 23$ and H1975 cells using a radioimmunoprecipitation assay lysis buffer (Thermo Fisher Scientific, Inc.). The protein concentration was determined using a BCA Protein Assay 
Table I. Association between miR-148a expression and clinicopathological characteristics in lung adenocarcinoma tissue.

\begin{tabular}{|c|c|c|c|c|}
\hline \multirow[b]{2}{*}{ Variables } & \multirow[b]{2}{*}{ Cases $(n=53)$} & \multicolumn{2}{|c|}{ miR-148a expression } & \multirow[b]{2}{*}{ P-value } \\
\hline & & Low $(n=25)$ & $\operatorname{High}(\mathrm{n}=28)$ & \\
\hline Sex & & & & 0.786 \\
\hline Male & 29 & 13 & 16 & \\
\hline Female & 24 & 12 & 12 & \\
\hline Age (years) & & & & 0.785 \\
\hline$<55$ & 22 & 11 & 11 & \\
\hline$\geq 55$ & 31 & 14 & 17 & \\
\hline Tumor size $(\mathrm{cm})$ & & & & 0.166 \\
\hline$<3$ & 23 & 8 & 15 & \\
\hline$\geq 3$ & 30 & 17 & 13 & \\
\hline Smoking history (years) & & & & 0.777 \\
\hline$<10$ & 20 & 11 & 9 & \\
\hline$\geq 10$ & 33 & 14 & 14 & \\
\hline Tumor differentiation & & & & 0.054 \\
\hline Well-moderate & 25 & 8 & 17 & \\
\hline Poor & 28 & 17 & 11 & \\
\hline TNM stage & & & & 0.006 \\
\hline I-II & 28 & 8 & 20 & \\
\hline III-IV & 25 & 17 & 8 & \\
\hline Lymph node metastasis & & & & 0.027 \\
\hline Negative & 24 & 7 & 17 & \\
\hline Positive & 29 & 18 & 11 & \\
\hline
\end{tabular}

TNM, Tumor, Node, Metastasis; miR, microRNA.

kit (Thermo Fisher Scientific, Inc.). Protein (50 $\mu \mathrm{g} / \mathrm{lane})$ was separated by $12 \%$ SDS-PAGE, which was then transferred onto a polyvinylidene fluoride membrane (Thermo Fisher Scientific, Inc.). The membrane was blocked with 5\% non-fat milk in TBST overnight at $4^{\circ} \mathrm{C}$. Following three washes with TBST, the membrane was incubated with rabbit anti-human E2F3 (cat. no. ab50917) and GAPDH (cat. no. ab9485; both 1:200; Abcam, Cambridge, MA, USA) primary antibodies at room temperature for $3 \mathrm{~h}$. Following three washes with TBST, the membrane was incubated with horseradish peroxidase-conjugated goat anti-rabbit secondary antibody (cat. no. ab6721; 1:5,000; Abcam) at room temperature for $1 \mathrm{~h}$. The protein signal was visualized using an enhanced chemiluminescence detection system (GE Healthcare, Chicago, IL, USA) and analysed with Quantity One ${ }^{\circledR}$ software (version 4.62; Bio-Rad Laboratories, Inc., Hercules, CA, USA). GAPDH was used as an internal control. The experiments were repeated three times.

Statistical analysis. Data are expressed as mean \pm standard deviation. SPSS software (version 19.0; IBM Corp., Armonk, NY, USA) was used to perform statistical analysis. The experiments were repeated three times. Differences between the groups were analysed using an unpaired Student's t-test or a paired Student's t-test. Differences between more than two groups were analysed using one-way analysis of variance with a Turkey's post hoc test. The Kaplan-Meier method and log-rank test was used for the survival analysis of patients grouped by high and low miR-148a expression. The association between miR-148a and the clinicopathological features in lung adenocarcinoma was analysed using a $\chi^{2}$ test. Pearson's correlation coefficient was used to analyse the correlation between the miR-148a and E2F3 expression levels in the lung adenocarcinoma tissues. $\mathrm{P}<0.05$ was considered to indicate a statistically significant difference.

\section{Results}

miR-148a is downregulated in lung adenocarcinoma. To examine the expression of miR-148a in lung adenocarcinoma, RT-qPCR was conducted. The data demonstrated that the expression of miR-148a was significantly reduced in lung adenocarcinoma tissues compared with that in adjacent non-tumour lung tissues (Fig. 1A). The association between miR-148a expression and clinicopathological characteristics were then studied in lung adenocarcinoma patients. Based on the mean expression value of miR-148a (1.57) in lung adenocarcinoma tissues, these lung adenocarcinoma patients were divided into the low expression and high expression groups. It was revealed that low miR-148a expression was significantly associated with advanced Tumor, Node, Metastasis (TNM) stages and lymph node metastasis of lung adenocarcinoma (Table I). 

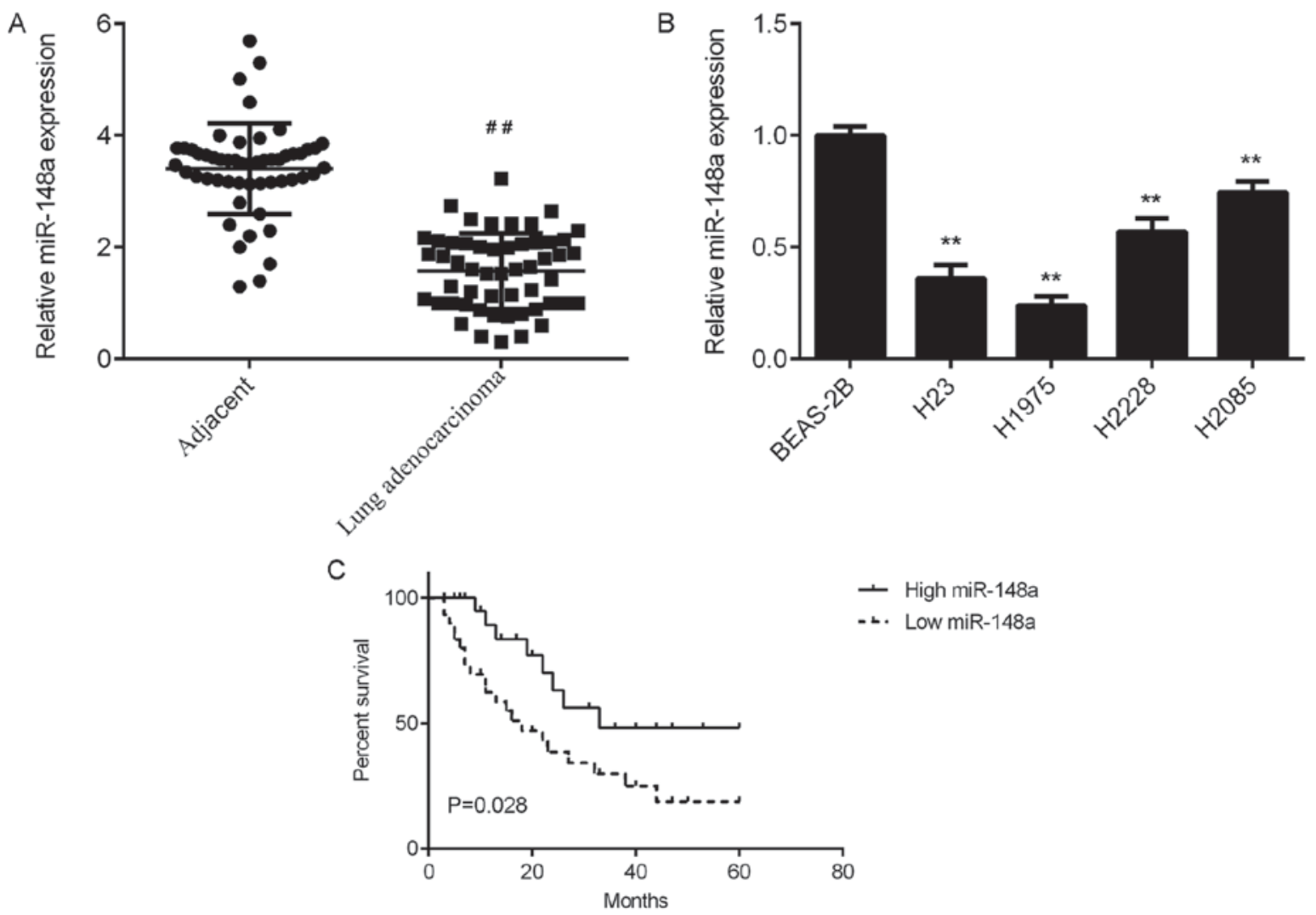

Figure 1. miR-148a is downregulated in lung adenocarcinoma. miR-148a expression was examined in (A) lung adenocarcinoma tissues, adjacent non-tumour lung tissues, (B) lung adenocarcinoma cell lines and a non-tumorigenic bronchial epithelium BEAS-2B cell line by reverse transcription-quantitative polymerase chain reaction. (C) The survival rate curves of the patients with low and high miR-148a expression. ${ }^{\# \#} \mathrm{P}<0.01 \mathrm{vs}$. Adjacent; ${ }^{* *} \mathrm{P}<0.01 \mathrm{vs}$. BEAS-2B. miR, microRNA.

Then, the miR-148a expression was examined in several common lung adenocarcinoma cell lines compared with normal bronchial epithelium BEAS-2B cells. The data indicated that miR-148a was also downregulated in these lung adenocarcinoma cell lines compared with BEAS-2B cells (Fig. 1B). Therefore, miR-148a is downregulated in lung adenocarcinoma cell lines, which may contribute to the malignant progression of this disease.

In addition, patients with low miR-148a expression had a shorter survival time compared with those with high miR-148a expression (Fig. 1C). These findings suggest that low miR-148a expression may predict poor prognosis for patients with lung adenocarcinoma.

miR-148a overexpression inhibits cell proliferation, colony formation and cell cycle progression of lung adenocarcinoma cells. As miR-148a was downregulated in lung adenocarcinoma cells, miR-148a mimics were transfected into H23 and H1975 cells to increase its expression. Following the transfection, RT-qPCR was conducted and the data revealed that miR-148a was significantly upregulated in H23 and H1975 cells in the miR-148a group compared with the control group (Fig. 2A). Additional experiments demonstrated that the overexpression of miR-148a significantly decreased the proliferation and colony formation of H23 and H1975 cells compared with the control group (Fig. 2B and C). Flow cytometry was then conducted to evaluate the cell cycle distribution and the data revealed that the upregulation of miR-148a caused a significant cell cycle arrest at the G1 stage compared with the control group (Fig. 2D). These results suggest that miR-148a overexpression inhibits the cell proliferation of the lung adenocarcinoma via inducing cell cycle arrest.

E2F3 is a target of miR-148a in lung adenocarcinoma cells. The underlying mechanisms of miR-148a in lung adenocarcinoma cells were investigated. Bioinformatic analysis was conducted to predict the putative target genes of miR-148a. As presented in Fig. 3A, E2F3 was a predicted target gene of miR-148a. To verify this prediction, a dual-luciferase reporter gene assay was performed in $\mathrm{H} 23$ and $\mathrm{H} 1975$ cells. The data indicated that overexpression of miR-148a in the miR-148a group significantly reduced the firefly luciferase activity of the WT 3'-UTR of E2F3 compared with that of the control group, but overexpression of miR-148a exhibited no significant effect on the luciferase activity of the MT 3'-UTR of E2F3 in H23 and H1975 cells (Fig. 3B and C). It was further observed that the overexpression of miR-148a significantly reduced the mRNA and protein expression of E2F3 in H23 and H1975 cells compared with the control group (Fig. 3D and E). Subsequently, H23 and H1975 cells were transfected with miR-148a inhibitor or NC inhibitor, respectively. Following transfection, the expression of miR-148a was significantly reduced in the miR-148a inhibitor group when compared with the NC inhibitor group (Fig. 3F). In addition, it was demonstrated that transfection with a miR-148a inhibitor significantly increased the mRNA and protein expression of 


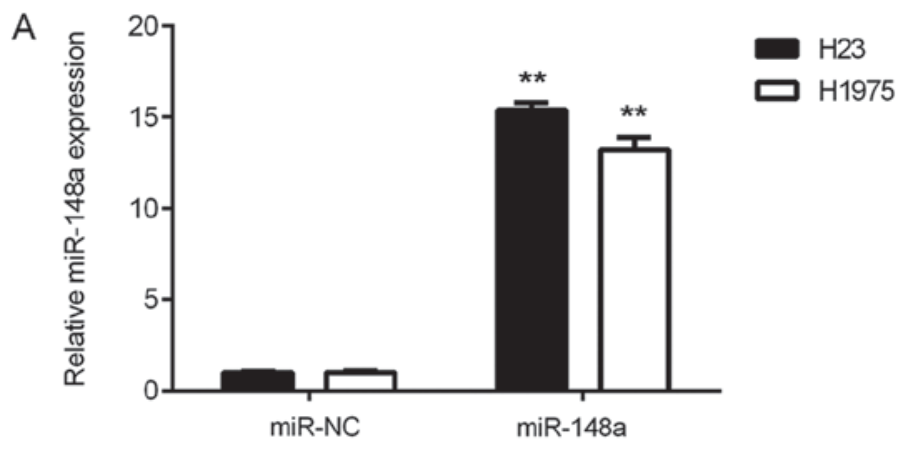

B

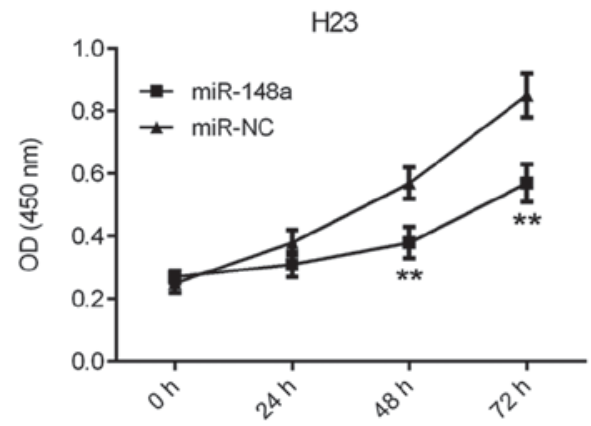

H1975

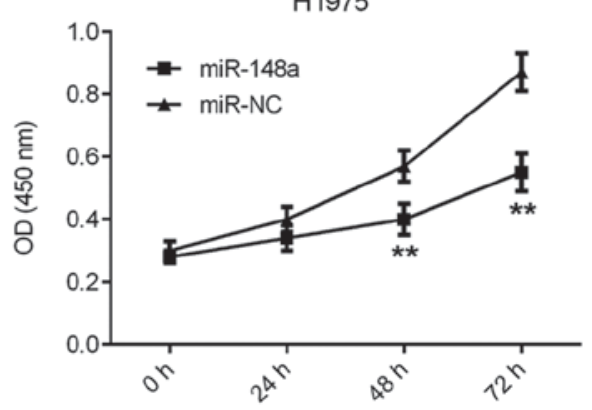

C

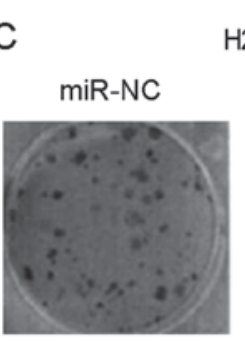

$\mathrm{H} 23$
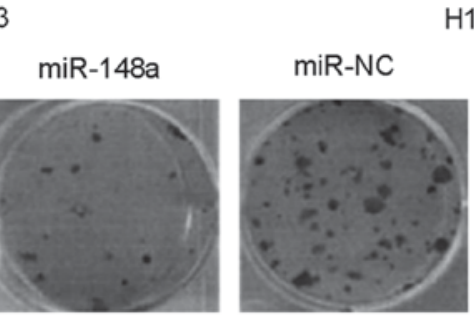

H1975
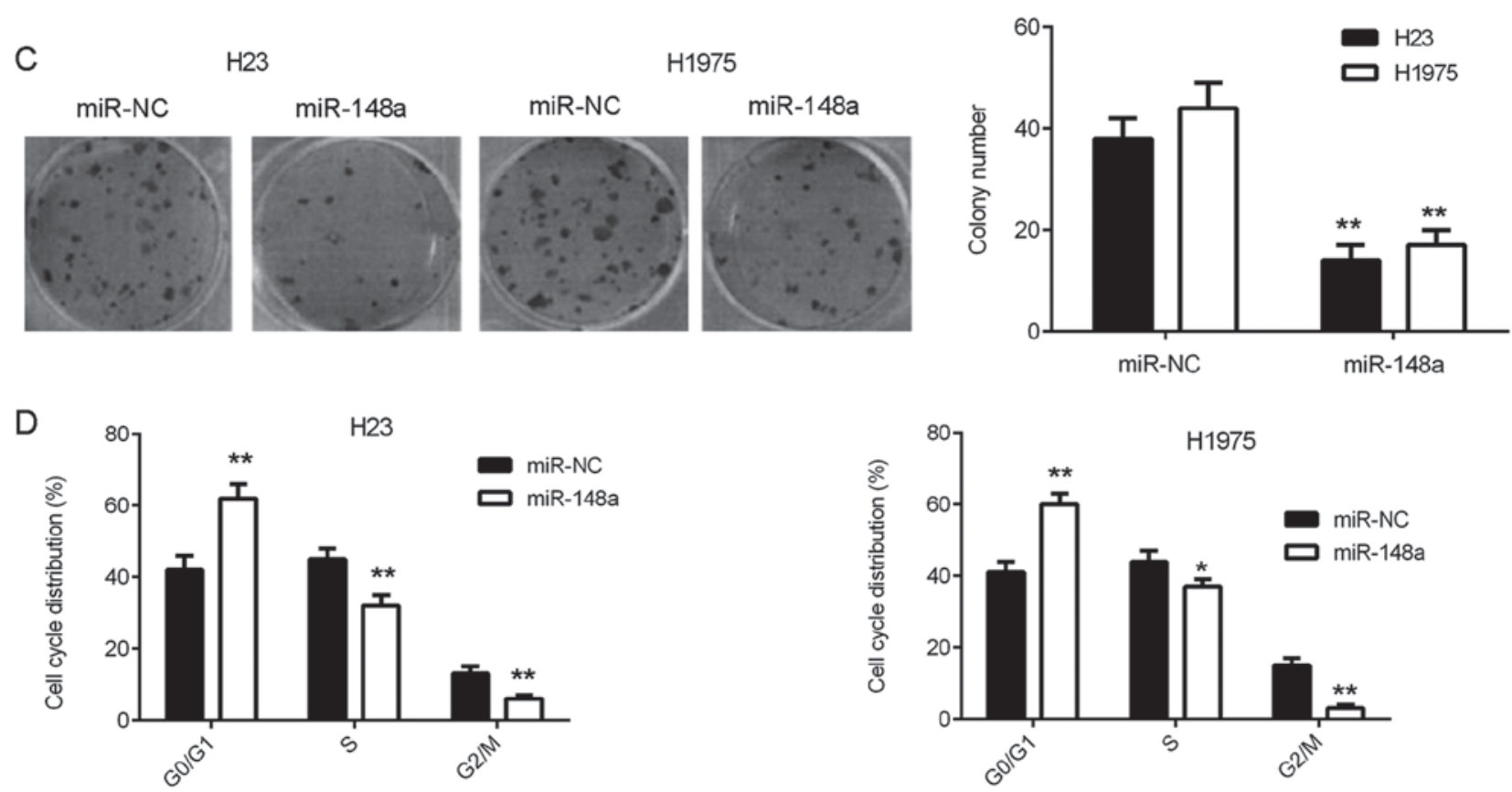

Figure 2. miR-148a overexpression inhibits cell proliferation of lung adenocarcinoma via inducing cell cycle arrest. miR-148a mimics or miR-NC was transfected into H23 and H1975 cells. (A) Reverse transcription-quantitative polymerase chain reaction was performed to examine the miR-148a expression. (B) Cell proliferation was determined using a Cell Counting kit- 8 assay. (C) Colony formation capacity. (D) Cell cycle distribution was determined using flow cytometry. ${ }^{*} \mathrm{P}<0.05$ and ${ }^{* *} \mathrm{P}<0.01$ vs. miR-NC. miR, microRNA; NC, negative control; OD, optical density.

E2F3 in H23 and H1975 cells compared with those in cells transfected with an NC inhibitor (Fig. 3G and H). Therefore, E2F3 was revealed to be a direct target of miR-148a and the expression of E2F3 was negatively mediated by miR-148a in lung adenocarcinoma cells.

E2F3 is upregulated in lung adenocarcinoma. RT-qPCR analyses were conducted to examine E2F3 mRNA expression in tissues, and the data indicated that E2F3 expression was significantly upregulated in lung adenocarcinoma tissues compared with that in adjacent non-tumour lung tissues (Fig. 4A). Then, the mRNA expression of E2F3 was examined in lung adenocarcinoma cell lines and BEAS-2B cells. The data indicated that E2F3 was also upregulated in lung adenocarcinoma cell lines compared with BEAS-2B cells (Fig. 4B). Notably, the expression of miR-148a was significantly and inversely correlated with E2F3 expression in lung adenocarcinoma tissues (Fig. 4C). Therefore, the increased expression of E2F3 may be caused by the reduced expression of miR-148a in lung adenocarcinoma tissues. 


\section{A}

\begin{tabular}{|c|c|c|c|c|c|c|c|}
\hline & $\begin{array}{l}\text { Predicted consequential pairing of target region (top) } \\
\text { and miRNA (bottom) }\end{array}$ & $\begin{array}{c}\text { Site } \\
\text { type }\end{array}$ & $\begin{array}{l}\text { Context+r } \\
\text { score }\end{array}$ & $\begin{array}{c}\text { Context }+\cdots \text { score } \\
\text { percentile }\end{array}$ & $\begin{array}{l}\text { Weighted } \\
\text { context+r score }\end{array}$ & $\begin{array}{c}\text { Conserved branch } \\
\text { length }\end{array}$ & h \\
\hline $\begin{array}{l}\text { Position 2037-2043 of E2F } 33 \text { UTR } 5 \\
\text { hsa-miR-148a-3p }\end{array}$ & 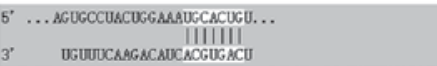 & $\begin{array}{c}7 \mathrm{mer}- \\
\mathrm{m} 8\end{array}$ & -0.24 & 90 & -0.24 & 6.250 & 32 \\
\hline
\end{tabular}

B

Wild-type of E2F3 3'UTR

5'...GGAAAUGCACUGU...3'

miR-148a

Mutant type of E2F3 3'UTR

3'...ACAUCACGUGACU...5'<smiles>c1cc2cc(c1)C2</smiles>

C

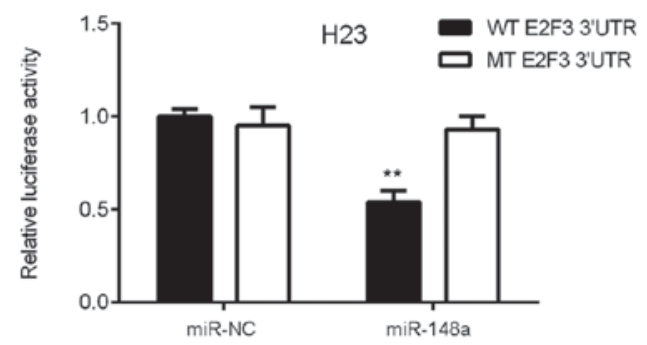

5'...GGAAAUGGUGAGU...3'

D
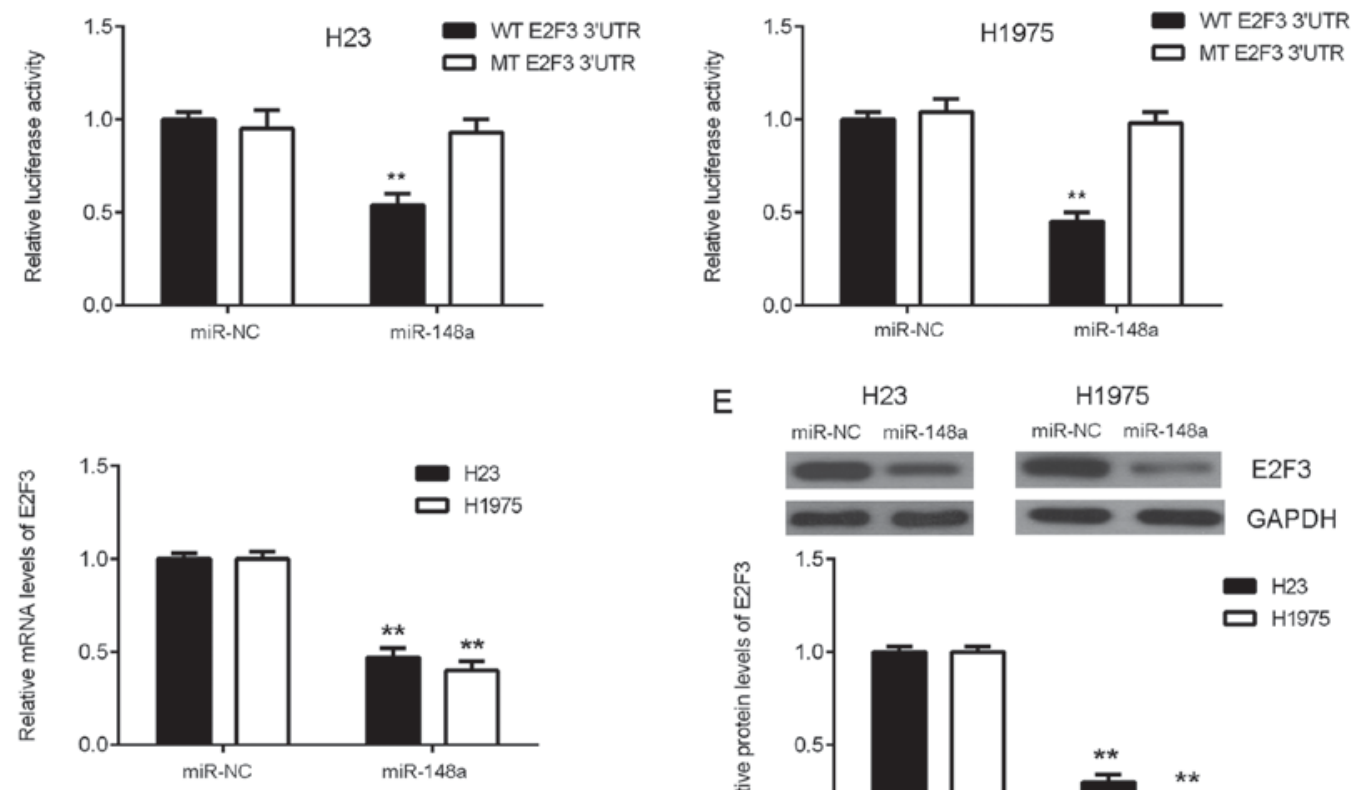

E

$\mathrm{H} 23$

H1975

miR-NC miR-148a miR-NC miR-148a

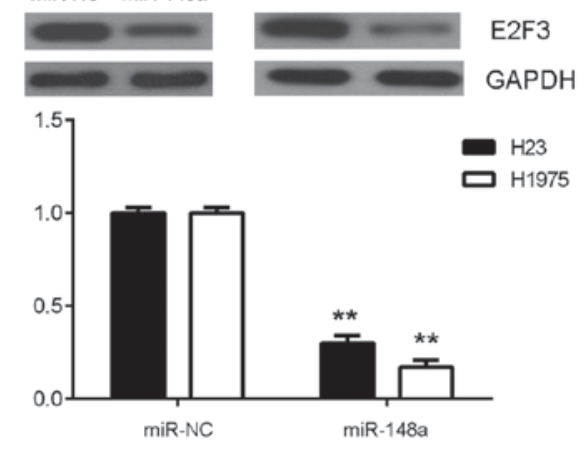

F

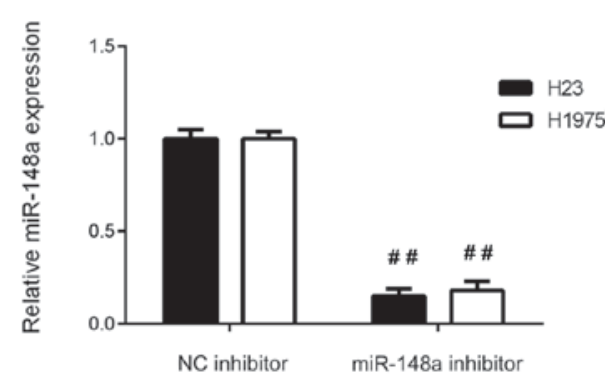

G

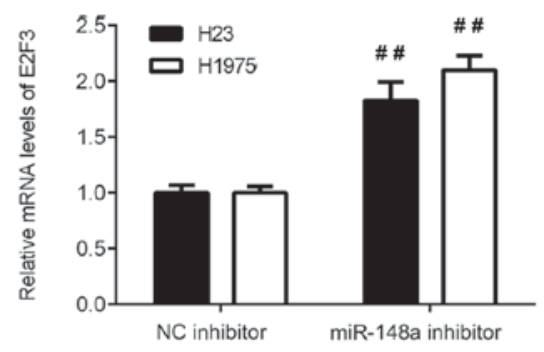

$\mathrm{H}$

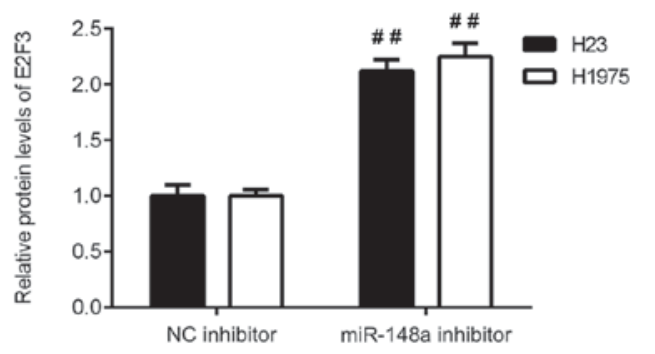

Figure 3. E2F3 is a target gene of miR-148a in lung adenocarcinoma. (A) Putative WT binding sequences in the 3'-UTR of E2F3. (B) Diagram of the putative WT and MT binding sequences in the 3'-UTR of E2F3. (C) H23 and H1975 cells were co-transfected with miR-148a mimics or miR-NC and WT-E2F3 or MT-E2F3 reporter gene plasmid, respectively. Following a 48-h transfection, relative luciferase activity was determined using a dual luciferase reporter assay system. The (D) mRNA and (E) protein expression of E2F3 was detected by RT-qPCR and western blot analyses in H23 and H1975 cells transfected with miR-148a mimics or miR-NC. (F) Transfection with miR-148 inhibitor significantly reduced the miR-148 expression compared with transfection with NC inhibitor. The $(\mathrm{G})$ mRNA and $(\mathrm{H})$ protein expression of E2F3 was detected by RT-qPCR and western blot analyses in H23 and H1975 cells transfected with miR-148a inhibitor or NC inhibitor. ${ }^{* *} \mathrm{P}<0.05$ vs. miR-NC. ${ }^{\# \#} \mathrm{P}<0.05$ vs. NC inhibitor. WT, wild type; MT, mutant type; UTR, untranslated region; miR, microRNA; NC, negative control; E2F3, transcription factor E2F3; RT-qPCR, reverse transcription-quantitative polymerase chain reaction. 

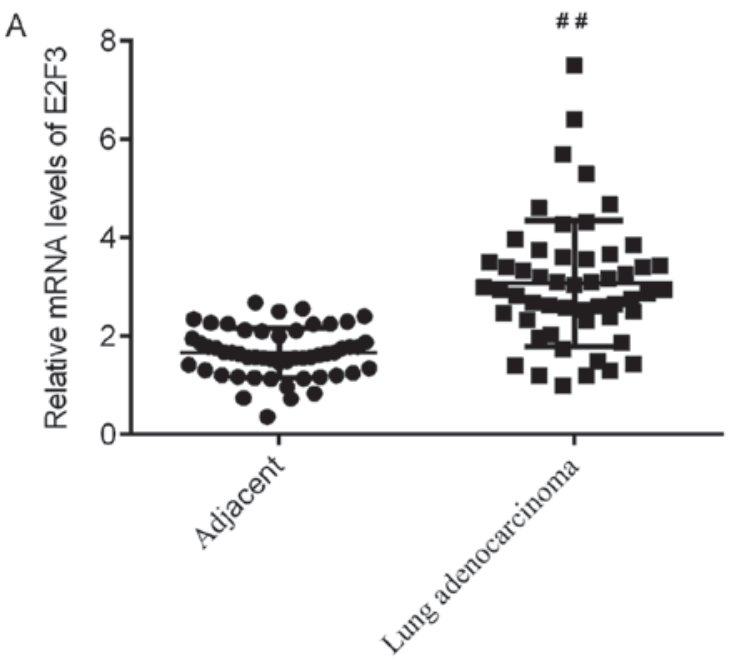

B
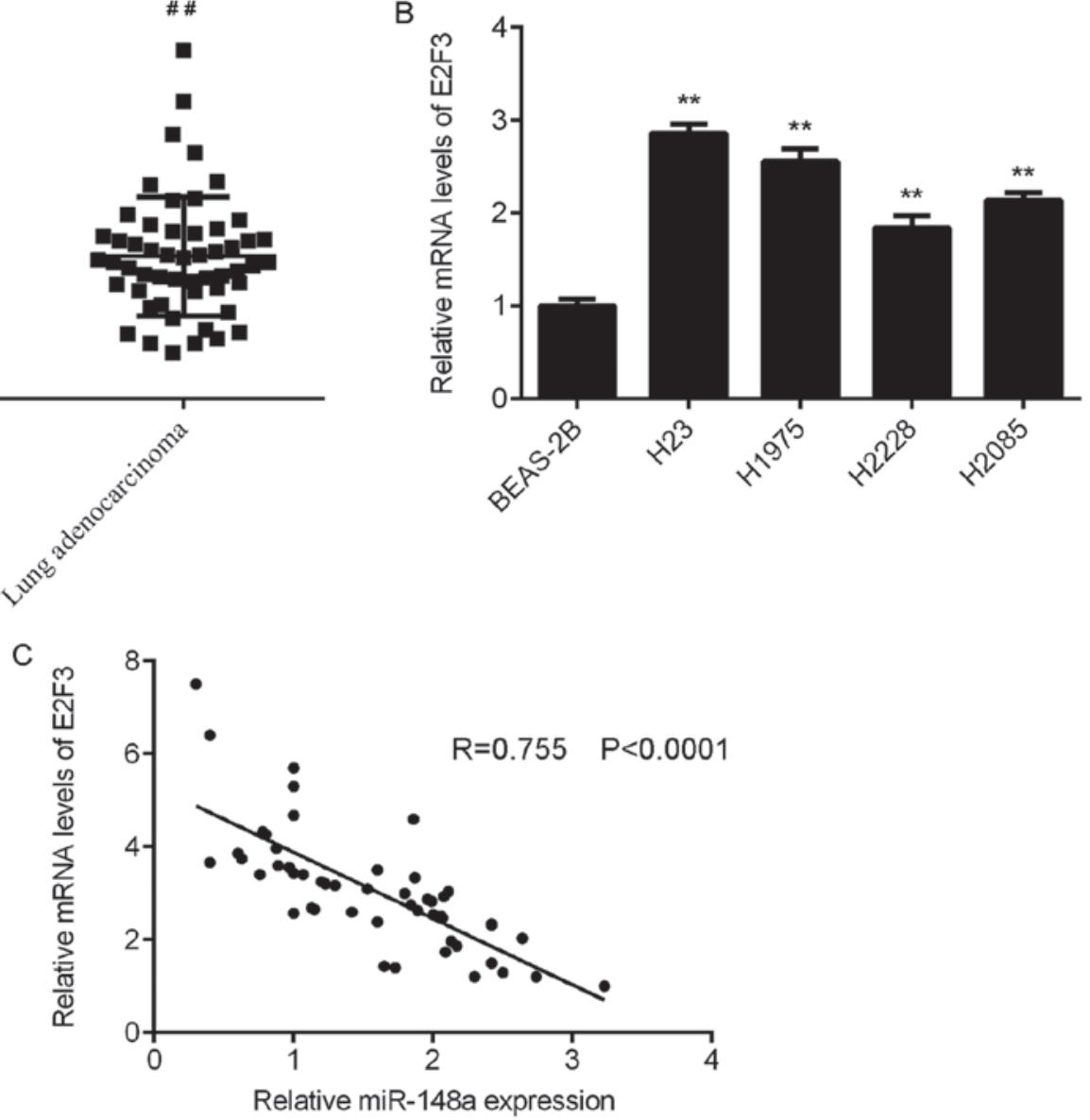

Figure 4. E2F3 is upregulated in lung adenocarcinoma. E2F3 mRNA expression was examined in (A) lung adenocarcinoma tissues, adjacent non-tumour lung tissues, (B) lung adenocarcinoma cell lines and a non-tumorigenic bronchial epithelium BEAS-2B cell line by reverse transcription-quantitative polymerase chain reaction. (C) The correlation of miR-148a expression to E2F3 mRNA expression in lung adenocarcinoma tissues. ${ }^{\# \#<0.01 ~ v s . ~ A d j a c e n t ; ~}$ ${ }_{* * *} \mathrm{P}<0.01$ vs. BEAS-2B. miR, microRNA; E2F3, transcription factor E2F3.

Restoration of E2F3 expression counteracts the suppressive effects of miR-148a overexpression on lung adenocarcinoma cells. The authors of the current study investigated whether the suppressive effects of miR-148a on lung adenocarcinoma cells were mediated by E2F3. H23 and H1975 cells were co-transfected with miR-148a mimics and the E2F3 expression or blank pcDNA3.1 plasmid. Following transfection, the E2F3 mRNA and protein expression levels were significantly and markedly increased, respectively, in the miR-148a+E2F3 group compared with the miR-148a+blank group (Fig. 5A and B). Subsequent functional assays revealed that the cell proliferation, colony formation and cell cycle progression were also significantly promoted in the miR-148a+E2F3 group compared with the miR-148a+blank group (Fig. 5C-E). These findings suggest that restoration of E2F3 expression counteracts the suppressive effects of miR-148a overexpression on lung adenocarcinoma cells.

\section{Discussion}

The present study aimed to examine miR-148a expression in lung adenocarcinoma and investigate the molecular mechanisms of miR-148a underlying lung adenocarcinoma growth. It was revealed that miR-148a was significantly downregulated in lung adenocarcinoma tissues and cell lines, and low miR-148a expression was significantly associated with advanced TNM stages and lymph node metastasis, as well as a shorter survival time for patients. Increasing miR-148a expression greatly decreased the cell proliferation, colony formation and cell cycle progression of H23 and H1975 cells. E2F3 was identified as a target of miR-148a in $\mathrm{H} 23$ and $\mathrm{H} 1975$ cells. In addition, the expression of E2F3 was negatively regulated by miR-148a in H23 and H1975 cells. E2F3 was greatly upregulated in lung adenocarcinoma tissues and cell lines, and the expression of miR-148a was inversely correlated with the E2F3 expression in lung adenocarcinoma tissues. Additional experiments demonstrated that increasing E2F3 expression counteracted the inhibitory effects on lung adenocarcinoma cells caused by miR-148a overexpression. These findings suggest that the downregulation of miR-148a may be implicated in the malignant progression of lung adenocarcinoma.

Deregulations of miR-148a expression have been observed in different cancer types (17-19). For example, miR-148a expression was downregulated in gastric cancer tissues compared with their corresponding non-tumour tissues, which was significantly associated with advanced TNM stages and lymph node metastasis, and induced the suppression of gastric cancer cell migration and invasion (20). In contrast, miR-148a is androgen-responsive and promotes prostate cell growth by inhibiting cullin-associated 
A

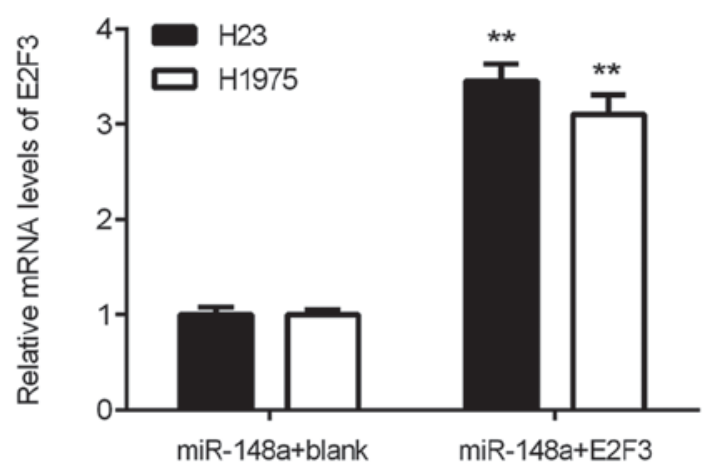

B

C
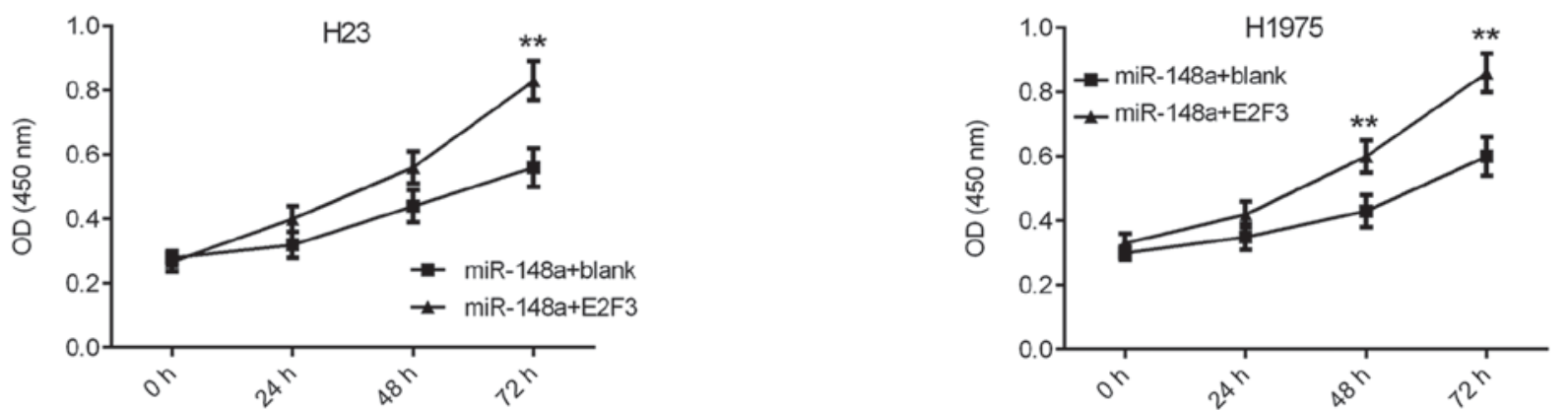

D $\mathrm{H} 23$
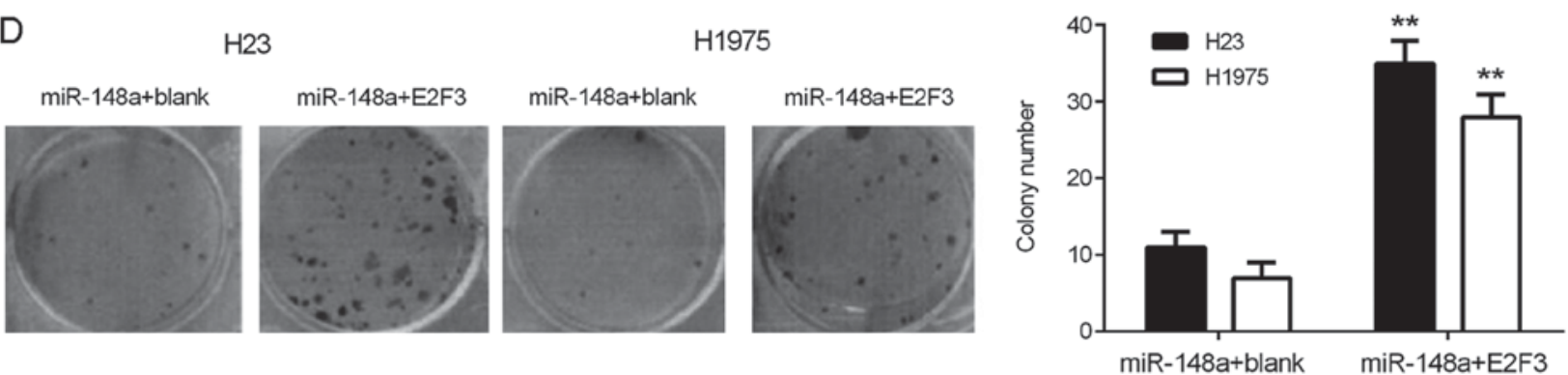

E

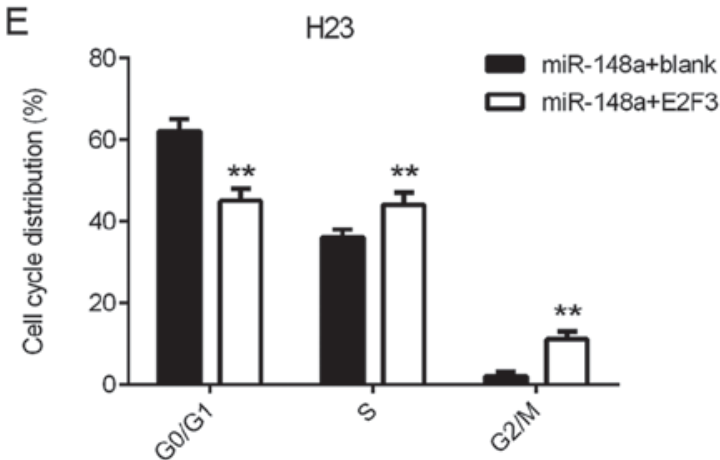

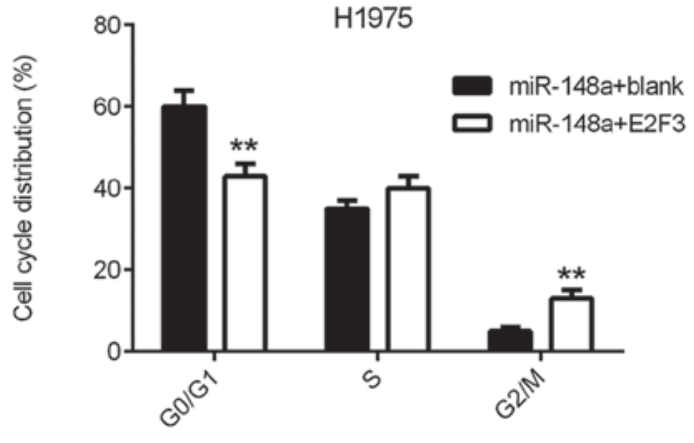

Figure 5. Restoration of E2F3 expression counteracts the suppressive effects of miR-148a overexpression on lung adenocarcinoma cells. H23 and H1975 cells were co-transfected with miR-148a mimics and pcDNA3.1-E2F3 expression or blank pcDNA3.1 plasmids. The (A) mRNA and (B) protein levels of E2F3 were examined using reverse transcription-quantitative polymerase chain reaction and western blot analyses. (C) Cell proliferation was determined using a Cell Counting kit-8 assay. (D) Colony formation capacity. (E) Cell cycle distribution was determined using flow cytometry. ${ }^{* *} \mathrm{P}<0.01$ vs. miR-148a+blank. E2F3, transcription factor E2F3; miR, microRNA; OD, optical density.

NEDD8-dissociated protein 1 expression (21). Reduced miR-148a expression contributed to the suppression of osteosarcoma cell death (22). These diverse functions of miR-148a in different cancer types may be due to the different tumour microenvironment.
Additionally, certain miR-148a targets have been identified, including receptor tyrosine-protein kinase erbB-3 (23), rho-associated protein kinase 1 (ROCK1) (20), ubiquitin carboxyl-terminal hydrolase 4 (19), DNA (cytosine-5)-methyltransferase 1 (24), proto-oncogene Wnt-1 (25), 
collagenase 3 (26) and CCK-BR (9). Li et al (27) demonstrated that miR-148a suppressed epithelial-mesenchymal transition by targeting ROCK1 in NSCLC cells. He and Xue (15) reported that miR-148a inhibited NSCLC cell proliferation and invasion via inhibition of STAT3 expression. In the current study, E2F3 was confirmed to be a direct target gene of miR-148a in lung adenocarcinoma cells. E2F3 is a key regulator of the G1/S phase transition, and the activation of E2F3 serves a role in promoting the development and progression of different cancer types through promoting the malignant phenotypes of cancer cells $(28,29)$. In addition, specific miRs have been identified as miRs that directly target E2F3 to inhibit cancer progression. For instance, miR-503 inhibits cell proliferation and induces apoptosis in colorectal cancer cells by targeting E2F3 (30). miR-200c inhibits the invasion, migration and proliferation of bladder cancer cells through inhibiting the expression of polycomb complex protein BMI-1 and E2F3 (31). In the present, it was revealed that E2F3 was significantly upregulated in lung adenocarcinoma tissues and cell lines, and the increased expression of E2F3 was inversely correlated with the reduced expression of miR-148a in lung adenocarcinoma tissues. These findings suggest that the downregulation of miR-148a contributes to the upregulation of E2F3 in lung adenocarcinoma. As further experiments demonstrated that the overexpression of E2F3 counteracted the inhibitory effects of miR-148a on lung adenocarcinoma cells, the authors of the present study suggest that targeting E2F3 may become a promising therapeutic strategy for lung adenocarcinoma.

In summary, the present study demonstrated that miR-148a has suppressive effects on the proliferation, colony formation and cell cycle progression of lung adenocarcinoma cells, at least in part, through directly targeting E2F3. Therefore, the authors of the current study suggest that miR-148a may be used as a potential candidate for the treatment of lung adenocarcinoma.

\section{Acknowledgements}

Not applicable.

\section{Funding}

No funding was received.

\section{Availability of data and materials}

All data generated or analysed during this study are included in this published article.

\section{Authors' contributions}

JL collected clinical tissues and wrote the manuscript. HT designed the study and revised the manuscript. LS and JL performed all experiments.

\section{Ethics approval and consent to participate}

The current study was approved by the Ethics Committee of Affiliated Hospital of Binzhou Medical College. All written informed consents have been obtained from patients involved in the current study.

\section{Patient consent for publication}

Not applicable.

\section{Competing interests}

The authors declare that they have no competing interests.

\section{References}

1. Siegel RL, Miller KD and Jemal A: Cancer statistics, 2015. CA Cancer J Clin 65: 5-29, 2015.

2. Torre LA, Bray F, Siegel RL, Ferlay J,Lortet-Tieulent J and Jemal A: Global cancer statistics, 2012. CA Cancer J Clin 65: 87-108, 2015.

3. Ambros V: The functions of animal microRNAs. Nature 431: 350-355, 2004.

4. Bartel DP: MicroRNAs: Genomics, biogenesis, mechanism, and function. Cell 116: 281-297, 2004.

5. Calin GA, Sevignani C, Dumitru CD, Hyslop T, Noch E, Yendamuri S, Shimizu M, Rattan S, Bullrich F, Negrini M and Croce CM: Human microRNA genes are frequently located at fragile sites and genomic regions involved in cancers. Proc Natl Acad Sci USA 101: 2999-3004, 2004.

6. Zhu Y, Zhao H, Rao M and Xu S: MicroRNA-365 inhibits proliferation, migration and invasion of glioma by targeting PIK3R3. Oncol Rep 37: 2185-2192, 2017.

7. Zheng Y, Lv X, Wang X, Wang B, Shao X, Huang Y, Shi L, Chen Z, Huang J and Huang P: MiR-181b promotes chemoresistance in breast cancer by regulating Bim expression. Oncol Rep 35: 683-690, 2016.

8. Liu S, Song L, Zeng S and Zhang L: MALAT1-miR-124-RBG2 axis is involved in growth and invasion of HR-HPV-positive cervical cancer cells. Tumour Biol 37: 633-640, 2016.

9. Yu B, Lv X, Su L, Wang B, Shao X, Huang Y, Shi L, Chen Z, Huang $J$ and Huang P: MiR-148a functions as a tumor suppressor by targeting CCK-BR via inactivating STAT3 and Akt in human gastric cancer. PLoS One 11: e0158961, 2016.

10. Xu X, Zhang Y, Jasper J, Lykken E, Alexander PB, Markowitz GJ, McDonnell DP, Li QJ and Wang XF: MiR-148a functions to suppress metastasis and serves as a prognostic indicator in triple-negative breast cancer. Oncotarget 7: 20381-20394, 2016.

11. Feng H, Wang Y, Su J, Liang H, Zhang CY, Chen X and Yao W: MicroRNA-148a suppresses the proliferation and migration of pancreatic cancer cells by down-regulating ErbB3. Pancreas 45: 1263-1271, 2016.

12. Li L, Chen YY, Li SQ, Huang C and Qin YZ: Expression of miR-148/152 family as potential biomarkers in non-small-cell lung cancer. Med Sci Monit 21: 1155-1161, 2015.

13. Joshi P, Jeon YJ, Lagana A, Middleton J, Secchiero P, Garofalo M and Croce CM: MicroRNA-148a reduces tumorigenesis and increases TRAIL-induced apoptosis in NSCLC. Proc Natl Acad Sci USA 112: 8650-8655, 2015.

14. Li J, Yu T, Cao J, Liu L, Liu Y, Kong HW, Zhu MX, Lin HC, Chu DD, Yao M and Yan MX: MicroRNA-148a suppresses invasion and metastasis of human non-small-cell lung cancer. Cell Physiol Biochem 37: 1847-1856, 2015.

15. He M and Xue Y: MicroRNA-148a suppresses proliferation and invasion potential of non-small cell lung carcinomas via regulation of STAT3. Onco Targets Ther 10: 1353-1361, 2017.

16. Livak KJ and Schmittgen TD: Analysis of relative gene expression data using real-time quantitative PCR and the 2(-Delta Delta $\mathrm{C}(\mathrm{T})$ ) method. Methods 25: 402-408, 2001.

17. Gailhouste L, Gomez-Santos L, Hagiwara K, Hatada I, Kitagawa N, Kawaharada K, Thirion M, Kosaka N, Takahashi RU, Shibata T, et al: miR-148a plays a pivotal role in the liver by promoting the hepatospecific phenotype and suppressing the invasiveness of transformed cells. Hepatology 58: 1153-1165, 2013.

18. Xia J, Guo X, Yan J and Deng K: The role of miR-148a in gastric cancer. J Cancer Res Clin Oncol 140: 1451-1456, 2014.

19. Heo MJ, Kim YM, Koo JH, Yang YM, An J, Lee SK, Lee SJ, Kim KM, Park JW and Kim SG: microRNA-148a dysregulation discriminates poor prognosis of hepatocellular carcinoma in association with USP4 overexpression. Oncotarget 5: 2792-2806, 2014.

20. Zheng B, Liang L, Wang C, Huang S, Cao X, Zha R, Liu L, Jia D, Tian Q, Wu J, et al: MicroRNA-148a suppresses tumor cell invasion and metastasis by downregulating ROCK1 in gastric cancer. Clin Cancer Res 17: 7574-7583, 2011. 
21. Murata T, Takayama K, Katayama S, Urano T, Horie-Inoue K, Ikeda K, Takahashi S, Kawazu C, Hasegawa A, Ouchi Y, et al: miR-148a is an androgen-responsive microRNA that promotes LNCaP prostate cell growth by repressing its target CAND1 expression. Prostate Cancer Prostatic Dis 13: 356-361, 2010.

22. Bhattacharya S, Chalk AM, Ng AJ, Martin TJ, Zannettino AC, Purton LE, Lu J, Baker EK and Walkley CR: Increased miR-155-5p and reduced miR-148a-3p contribute to the suppression of osteosarcoma cell death. Oncogene 35: 5282-5294, 2016.

23. Yu J,Li Q, Xu Q, Liu L and Jiang B: MiR-148a inhibits angiogenesis by targeting ERBB3. J Biomed Res 25: 170-177, 2011.

24. Long XR, He Y, Huang C and Li J: MicroRNA-148a is silenced by hypermethylation and interacts with DNA methyltransferase 1 in hepatocellular carcinogenesis. Int J Oncol 44: 1915-1922, 2014.

25. Jiang Q, He M, Ma MT, Wu HZ, Yu ZJ, Guan S, Jiang LY, Wang Y, Zheng DD, Jin F and Wei MJ: MicroRNA-148a inhibits breast cancer migration and invasion by directly targeting WNT-1. Oncol Rep 35: 1425-1432, 2016.

26. Xue J, Chen Z, Gu X, Zhang Y and Zhang W: MicroRNA-148a inhibits migration of breast cancer cells by targeting MMP-13. Tumour Biol 37: 1581-1590, 2016.
27. Li J, Song Y, Wang Y, Luo J and Yu W: MicroRNA-148a suppresses epithelial-to-mesenchymal transition by targeting ROCK1 in non-small cell lung cancer cells. Mol Cell Biochem 380: 277-282, 2013.

28. Noguchi S, Mori T, Otsuka Y, Yamada N, Yasui Y, Iwasaki J, Kumazaki M, Maruo K and Akao Y: Anti-oncogenic microRNA-203 induces senescence by targeting E2F3 protein in human melanoma cells. J Biol Chem 287: 11769-11777, 2012.

29. Xiao F, Zhang W, Chen L, Chen F, Xie H, Xing C, Yu X, Ding S, Chen K, Guo H, et al: MicroRNA-503 inhibits the G1/S transition by downregulating cyclin D3 and E2F3 in hepatocellular carcinoma. J Transl Med 11: 195, 2013.

30. Chang SW, Yue J, Wang BC and Zhang XL: miR-503 inhibits cell proliferation and induces apoptosis in colorectal cancer cells by targeting E2F3. Int J Clin Exp Pathol 8: 12853-12860, 2015.

31. Liu L, Qiu M, Tan G, Liang Z, Qin Y, Chen L, Chen H and Liu J: miR-200c Inhibits invasion, migration and proliferation of bladder cancer cells through down-regulation of BMI-1 and E2F3. J Transl Med 12: 305, 2014. 